\title{
Nosocomial infections increase the length of hospital stay(Letter)
}

- Carpio-Zevallos, M.S.a.Email Author,

- Sobrado-Jara, K.S.aEmail Author,

- Carreazo, N.Y.'Email Author

- View Correspondence (jump link)

- aUniversidad Peruana de Ciencias Aplicadas, Calle Pradilla 180, San Borja, Lima 11, Peru

- $\quad$ bServicio de Cuidados Intensivos Pediátricos, Hospital de Emergencias Pediátricas, Escuela de Medicina, Universidad Peruana de Ciencias Aplicadas, Garzon 685, Lima 11, Peru

Abstract View references (3)

[No abstract available]

\section{Indexed keywords}

- ISSN: 24054577

- Source Type: Journal

- Original language: English

- DOI: 10.1016/j.clnesp.2018.12.086

- PubMed ID: 30904225

- Document Type: Letter

- Publisher: Elsevier Ltd 